\title{
Confirmation of ring protons of 2-((3-(trifluoromethyl) phenyl) amino) nicotinic acid using correlation spectroscopy NMR technique.
}

\author{
Prashant Dwivedi ${ }^{1}$, Kartikeya Dwivedi ${ }^{2}$, Manish Mitra ${ }^{3}$ \\ ${ }_{1}$ Noida International University; Greater Noida; (U.P) \\ ${ }_{2}$ Maharaj Singh College; Saharanpur (U.P) \\ ${ }_{3}$ United College of Engineering \& Research; Greater Noida, (U.P)
}

\begin{abstract}
The power of Nuclear Magnetic Resonance spectroscopy (NMR) in structure elucidation derives in large part from its ability to establish bonding connectivity (via J-coupling interaction) or through space proximity (via dipolar coupling interactions) of nuclei. The amount of time consumed in elucidating a structure depends on the rate at which these interaction can be detected by NMR and analyzed.1D NMR methods most often explore interactions between only few nuclei at a time: spin-spin decoupling measurements are used to demonstrate through-bond connectivity; and NOE measurements are used to probe inter-nuclear distances, 2Dimensional NMR experiments provide much more structural information in a given time period. Correlation spectroscopy was used to elucidate the aromatic proton position in NMR spectra.
\end{abstract}

Keywords: NMR, J-coupling, dipolar coupling, NOE, 2 dimensional NMR.

\section{Introduction}

The key point in all this is that magnetisation transfer occurs between coupled spins. To appreciate the outcome of this in the final COSY spectrum, consider the case of two J-coupled spins, A and X, with a coupling constant of $J_{A X}$ and chemical shift offsets of $\sqrt{A}_{A}$ and $\sqrt{ } X$. The magnetisation associated with spin a will, after the initial $90^{0}$ pulse, precess during t 1 according to its chemical shift offset, $\sqrt{A}_{\mathrm{A}}$. The second $90^{\circ}$ pulse then transfers some part of this magnetisation to the coupled $\mathrm{X}$ spin, whilst some remains associated with the original spin $\mathrm{A}$. That which remains with $A$ will then precess in the detection period at a frequency $\sqrt{ } \mathrm{A}$ just as it did during $\mathrm{t} 1$, so in the final spectrum, will produce a peak at $\sqrt{A}_{A}$ in both dimensions, denoted $\left(V_{A}, V_{A}\right)$. This peak is therefore equivalent to that observed for the uncoupled AX system and because it represents the same frequency in both dimensions, it sits on the diagonal of the $2 \mathrm{D}$ spectrum and is therefore referred to as a diagonal peak. In contrast, the transferred magnetisation will precess in $\mathrm{t}_{2}$ at the frequency of the new 'host' spin $\mathrm{X}$ and will thus produce a peak corresponding to two different chemical shifts in the two dimensions $\left(V_{A}, V_{X}\right)$. This peak sits away from the diagonal and is therefore referred to as an off-diagonal or, more commonly, a crosspeak This is the peak of interest as it provides direct evidence of coupling between spins A and X. The whole process operates in the reverse direction also, that is, the same arguments apply for magnetisation originally associated with the $\mathrm{X}$ spin, giving rise to a diagonal peak at $\left(V_{x}, V_{x}\right)$ and a crosspeak at $\left(V_{x}, V_{A}\right)$. Thus, the COSY spectrum is symmetrical about the diagonal, with crosspeaks on either side of it mapping the same interaction.

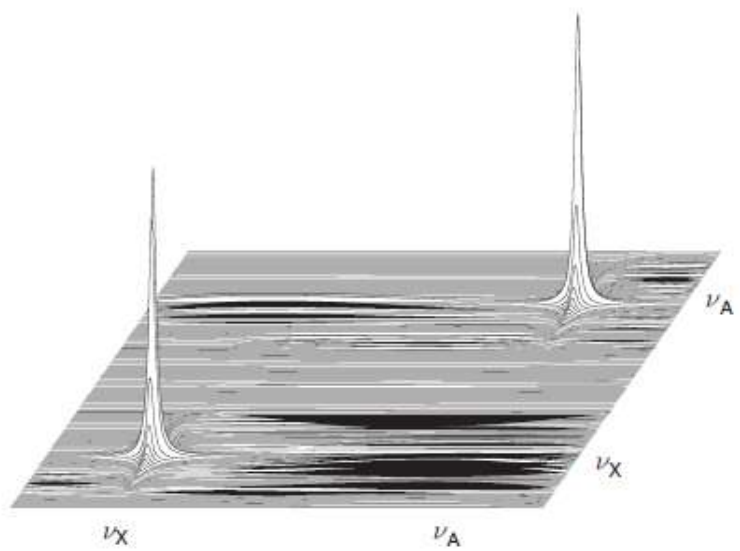

Figure-1: Sample containing two uncoupled spins, $A$ and $X$, of offsets $\sqrt{A}_{A}$ and $\sqrt{X}_{X}$. Each produces a 2D peak at its corresponding chemical shift offset in both dimensions. 


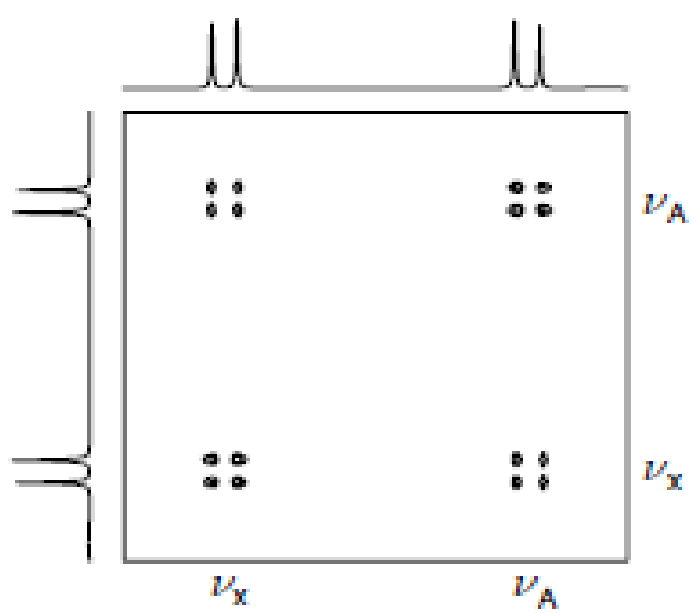

Figure-2 : The COSY spectrum of a coupled, two-spin AX system. Diagonal peaks are equivalent to those observed in the 1D spectrum whilst crosspeaks provide evidence of a coupling between the correlated spins.

Theory of polarisation transfer in the INEPT experiment, it was shown that the basic requirement for the transfer of polarization was an anti-phase disposition of the doublet vectors of the source spin, which for INEPT was generated by a spin-echo sequence. Magnetisation components that were in-phase just before the second $90^{\circ}$ pulse would not contribute to the transfer, hence the $\Delta$ period was optimised to maximise the anti-phase component. The same condition applies for magnetisation transfer between two protons as in the COSY experiment. This requires that the proton-proton coupling be allowed to evolve to give a degree of anti-phase magnetisation that may be transferred by the second pulse, whilst the in-phase component remains associated with the original spin. The coupling evolution period for COSY is the $t_{1}$ period so that the amount of transferred magnetisation detected in $t_{2}$ is also modulated as a function of $t_{1}\left(\sin 180 \mathrm{Jt}_{1}\right)$. Likewise, the amplitude of the inphase, non-transferred component is also modulated in $\mathrm{t}_{1}$ by the coupling $\left(\cos 180 \mathrm{Jt}_{1}\right)$, and this produces the coupling fine structure of the diagonal peak in $\mathrm{f}_{1}$.
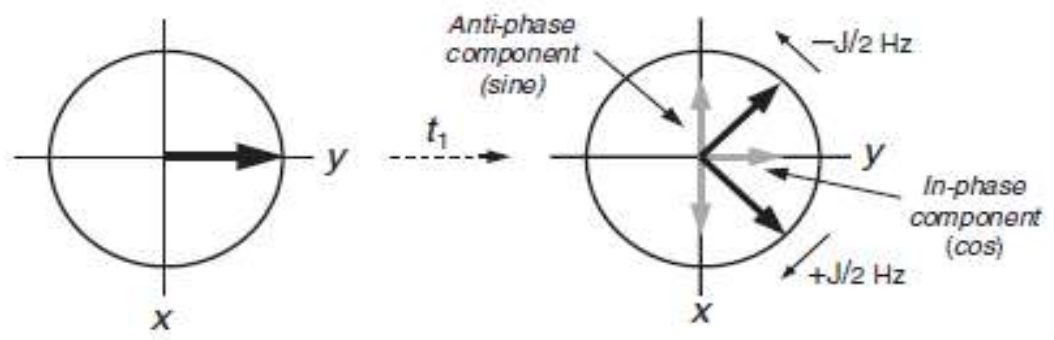

Figure-3: Coupling evolution during $\mathrm{t}_{1}$ produces in-phase and antiphase magnetisation components. Only the anti-phase component contributes to magnetisation transfer and hence to crosspeaks in the 2D spectrum.

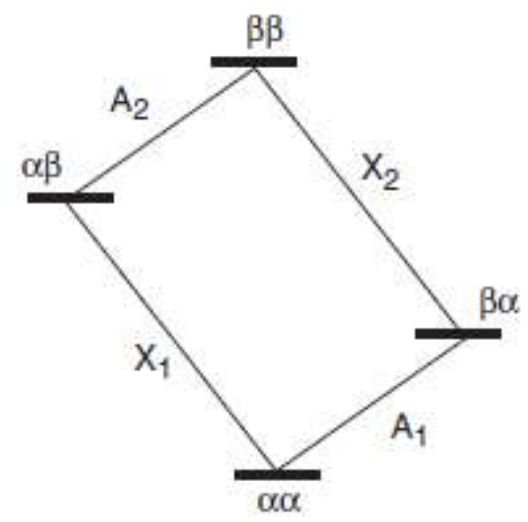

Figure-4: A schematic energy level diagram for the coupled two spins AX system.

As for $1 \mathrm{D}$ data, $\mathrm{f}_{1}$ quadrature detection requires two data sets that differ in phase by $90^{\circ}$ to be collected, thus providing the necessary sine and cosine amplitude-modulated data. Since thef $f_{1}$ dimension is generated 
artificially, there is strictly no reference rf to define signal phases, so itis the phase of the pulses that bracket $t_{1}$ that dictate the phase of the detected signal. Thus, foreach $t_{1}$ increment, two data sets are collected, one with a $90_{x}$ preparation pulse $\left(\mathrm{t}_{1}\right.$ sine modulation) and the other with $90_{\mathrm{y}}\left(\mathrm{t}_{1}\right.$ cosine modulation), both stored separately These two sets are then equivalent to the two channel data collected with simultaneous acquisition, which produces the desired frequency discrimination when subject to a complex FT (also referred to as a hypercomplex transform in relation to $2 \mathrm{D}$ data). The rate of sampling in $t_{1}$ or, in other words, the size of the $t_{1}$ time increment, is dictated by the $f_{1}$ spectral width and is subject to the same rules as for the simultaneous sampling of one-dimensional data. This method is derived from the original work of States, Haberkorn and Ruben and is therefore often referred to in the literature as the States method of $\mathrm{f}_{1}$ quad detection.
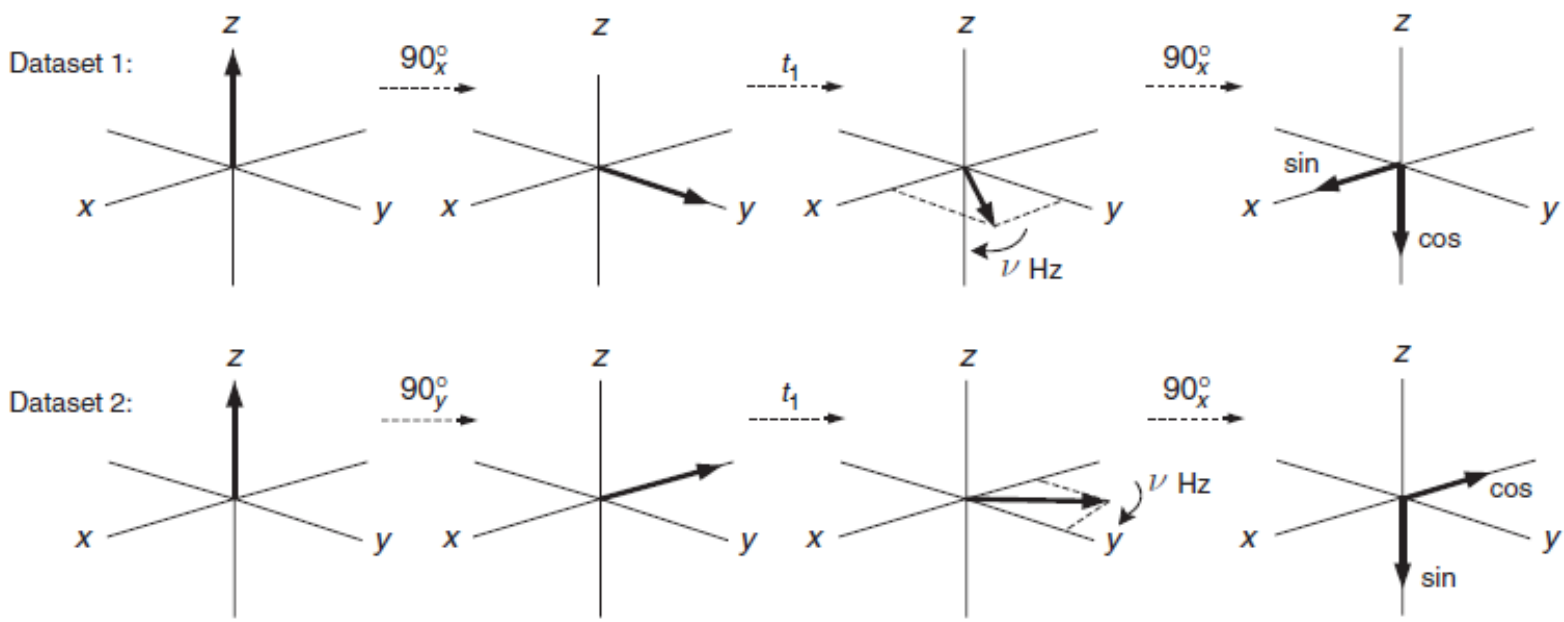

Figure-5 : The States method of $f_{1}$ quadrature detection requires two data sets to be acquired per increment to generate separate sine- and cosine modulated data sets.

\section{Methodology}

The success of any NMR experiment is, of course, crucially dependent on the correct setting of the acquisition parameters. In the case of 2D experiments, one has to consider the parameters for each dimension separately, and we shall see that the most appropriate parameter settings for $\mathrm{f}_{2}$ are rarely optimum for $\mathrm{fl}$. Likewise, one has to give rather more thought to the setting up of a 2D experiment than is usually required for 1D acquisitions to make optimum use of the instrument time available and data storage space. spectral widths, which should be the same in both dimensions of the COSY experiment, should be kept to minimum values with transmitter offsets adjusted so as to retain only the regions of the spectrum that will provide useful correlations. It is usually possible to reduce spectral widths to well below the 10-ppm or so proton window observed in 1D experiments. The use of excessively large windows leads to poorer digital resolution in the final spectrum or requires greater data sizes, neither of which is desirable. The spectral widths in turn define the sampling rates for data in $t_{2}$, in exact analogy with $1 D$ acquisitions, and the size of the $t_{1}$ increment, again according to the Nyquist criteria. The acquisition time $\left(A Q_{t}\right)$, and hence the digital resolution $\left(1 / \mathrm{AQ}_{\mathrm{t}}\right)$, for each dimension is then dictated by the number of data points collected in each. For $t_{2}$, this is the number of data points digitised in each FID, whilst for $t_{1}$ this is the number of FIDs collected over the course of the experiment. The appropriate setting of these parameters is a most important aspect to setting up a 2D experiment, and the way in which one thinks about acquisition times and digital resolution in a $2 \mathrm{D}$ data set is, necessarily, quite different from that in a 1D experiment. As an illustration, imagine transferring the typical parameters used in a $1 \mathrm{D}$ proton acquisition into the two dimensions of COSY. The acquisition time might be $4 \mathrm{~s}$, corresponding to a digital resolution of 0.25 $\mathrm{Hz} / \mathrm{pt}$, with no relaxation delay between scans. On, for example, a 400-MHz instrument, with a 10 ppm spectral width, this digital resolution would require $32 \mathrm{~K}$ words to be collected per FID. The 2D equivalent, with States quad detection in $f_{1}$ and with axial-peak suppression, requires four scans to be collected for each $t_{1}$ increment. The mean acquisition time for each would be $6 \mathrm{~s}$ ( $\mathrm{t}_{2}$ plus the mean $\mathrm{t}_{1}$ value), corresponding to $24 \mathrm{~s}$ of data collection per FID. If $16 \mathrm{~K} \mathrm{t} 1$ increments were to be made for the $\mathrm{f} 1$ dimension (two data sets are collected for each $t_{1}$ increment remember), this would correspond to a total experiment time of about 4.5 days. Furthermore, the size of the resulting data matrix would be a little over 1000 million words, and with a typical 32-bit-perword computer system, this requires some 4GB of disk space! We will agree that 4 days for a basic COSY acquisition is quite unacceptable, let alone the need for such disk space per experiment; so acquiring data with such high levels of digitisation in both dimensions is clearly not possible. 
The key lies in deciding on what level of digitisation is required for the experiment in hand. The first point to notice is that adding data points to extend the $t_{2}$ dimension leads to a relatively small increase in the overall length of the experiment, so we may be quite profligate with these (although they will lead to a corresponding increase in the size of the data matrix). Moreover, adding $\mathrm{t} 1$ data points requires that a complete FID of potentially many scans is required per increment, which makes a far greater increase to the total data collection time. Thus, one generally aims to keep the number of $t_{1}$ increments to a minimum, which is consistent with resolving the correlations of interest and increasing $t_{2}$ as required when higher resolution is necessary. For this reason, the digital resolution in $\mathrm{f}_{2}$ is often greater than that in $\mathrm{f}_{1}$, particularly in the case of phase-sensitive data sets. The use of smaller $\mathrm{AQt}_{1}$ is, in general, also preferred for reasons of sensitivity since FIDs recorded for longer values of $t_{1}$ will be attenuated by relaxation and so will contribute less to the overall signal intensity. The use of small $\mathrm{AQt}_{1}$ is likely to lead to truncation of the $\mathrm{t}_{1}$ data, and it is then necessary to apply suitable window functions that force the end of the data to zero to reduce the appearance of truncation artefacts.

For COSY in particular, one of the factors that limits the level of digitisation that can be used is the presence of intrinsically anti-phase crosspeaks, since too low a digitisation will cause these to cancel and the correlation to disappear The level of digitisation will also depend on the type of experiment and the data one expects to extract from it. For absolute-value COSY, one is usually interested in establishing where correlations exist, with little interest in the fine structure within these crosspeaks. In this case, it is possible to use a low level of digitisation consistent with identifying correlations. As a rule of thumb, a digital resolution of $\mathrm{J}$ to $2 \mathrm{~J} \mathrm{~Hz} / \mathrm{pt}$ (AQ of $1 / \mathrm{J}$ to $1 / 2 \mathrm{~J} \mathrm{~s}$ ) should enable the detection of most correlations arising from couplings of $\mathrm{J} \mathrm{Hz}$ or greater. Thus for a lower limit of, say, $3 \mathrm{~Hz}$, a digital resolution of $3-6 \mathrm{~Hz} / \mathrm{pt}$ (AQ of $\sim 300-150 \mathrm{~ms}$ ) will suffice. The $\mathrm{AQ}_{\mathrm{tI}}$ is typically half that for $\mathrm{t} 2$ in this experiment, with one level of zero- filling applied in $\mathrm{t} 1$ so that the final digital resolution is the same in both dimensions of the spectrum (as required for symmetrisation).

For phase-sensitive data acquisitions, one is likely to be interested in using the information contained within the crosspeak multiplet structures, and a higher degree of digitisation is required to adequately reflect this, a more appropriate target being around $\mathrm{J} / 2 \mathrm{~Hz} / \mathrm{pt}$ or better (AQ of $2 / \mathrm{J} \mathrm{s}$ or greater). Again, digitisation in $\mathrm{t}_{2}$ is usually two or even four or eight times greater than that in $t 1$. In either dimension, but most often in $t_{1}$, this may be improved by zero-filling, although one must always remember that it is the length of the time-domain acquisition that places a fundamental limit on peak resolution and the effective linewidths after digitisation, regardless of zero-filling. The alternative approach for extending the time domain data is to use forward linear prediction when processing the data. The rule as ever is that high resolution requires long data-sampling periods.

Having decided on suitable digitisation levels and data sizes, one is left to choose the number of scans or ransients to be collected per FID and the repetition rates and hence relaxation delays to employ. The minimum number of transients is dictated by the minimum number of steps in the phase cycle used to select the desired signals. Further scans may include additional steps in the cycle to suppress artefacts arising from imperfections. Beyond this, further transients should be required only for signal averaging when sensitivity becomes a limiting factor. Since most experiments are acquired under 'steady-state' conditions, it is also necessary to include 'dummy' scans prior to data acquisition to allow the steady state to establish. On modern instruments that utilize double buffering of the acquisition memory, dummy scans are required only at the very beginning of each experiment to make a negligible increase to the total time required. On older instruments that lack this feature, it is necessary to add dummy scans for each $t_{1}$ increment, and these may then make a significant contribution to the total duration of the experiment. The repetition rate will depend upon the proton $\mathrm{T}_{1} \mathrm{~S}$ in the molecule, and since the sequence uses $90^{\circ}$ pulses, the optimum sensitivity is achieved by repeating every $1.3 \mathrm{~T}_{1} \mathrm{~s}$.

Returning to the example of $400 \mathrm{MHz}$ acquisition discussed above, we can apply more appropriate criteria to the selection of parameters. Table 1 compares the result from above with more realistic data, and it is clear that under these conditions, COSY becomes a viable experiment, requiring only hours or even minutes to collect, rather than many days. The introduction of PFGs to high-resolution spectroscopy allows experiments to be acquired with only one transient per FID where sensitivity is not limiting, thus further reducing the total time required for data collection. The data storage requirements in these realistic examples are also well within the capabilities of modern computing hardware and are likely to become increasingly less significant as this develops further. 
Table-1: Illustrative data tables for COSY experiments

\begin{tabular}{lccccccc}
\hline Experiment & Spectral width/ppm & $\mathrm{N}\left(t_{2}\right)$ & $\mathrm{N}\left(t_{1}\right)$ & $\mathrm{Hz} / \mathrm{pt}\left(t_{2}\right)$ & $\mathrm{Hz} / \mathrm{pt}\left(t_{1}\right)$ & Experiment time & Raw data-set size \\
\hline (a) Phase-sensitive & $10 \times 10$ & $32 \mathrm{~K}$ & $32 \mathrm{~K}$ & 0.25 & 0.25 & 4.5 days & $1000 \mathrm{M}$ words \\
(b) Phase-sensitive & $6 \times 6$ & $2 \mathrm{~K}$ & $\mathrm{IK}$ & 2.3 & 2.3 & 55 min & $2 \mathrm{M}$ words \\
(c) Absolute value & $6 \times 6$ & $1 \mathrm{~K}$ & 256 & 4.6 & 4.6 & $22 \mathrm{~min}$ & $0.25 \mathrm{M}$ words \\
\hline
\end{tabular}

Scenario (a) transplants acquisition parameters from a typical 1D proton spectrum into the second dimension leading to unacceptable time requirements, whereas (b) and (c) use parameters more appropriate to 2D acquisitions. All calculations use phase cycles for f1 quad detection and axial-peak suppression only and for (b) and (c), a recovery delay of $1 \mathrm{~s}$ between scans. A single zero-filling in $\mathrm{f} 1$ was also employed for (b) and (c).

\section{Results \& Discussion}

For the current study sample of 2-((3-(trifluoromethyl)phenyl)amino)nicotinic acid was taken for the analysis and than the data was interpreted using the correlation spectroscopy NMR technique. Proton NMR data was acquired in DMSO solvent and the result was found to be :dd( 1H;6.95); d(1H;7.34); d(1H;7.87); t(1H;7.53); dd(1H;8.29); dd(1H;8.45); s(1H;8.31); s(1H;10.65); $\mathrm{s}(1 \mathrm{H} ; 13.71)$.
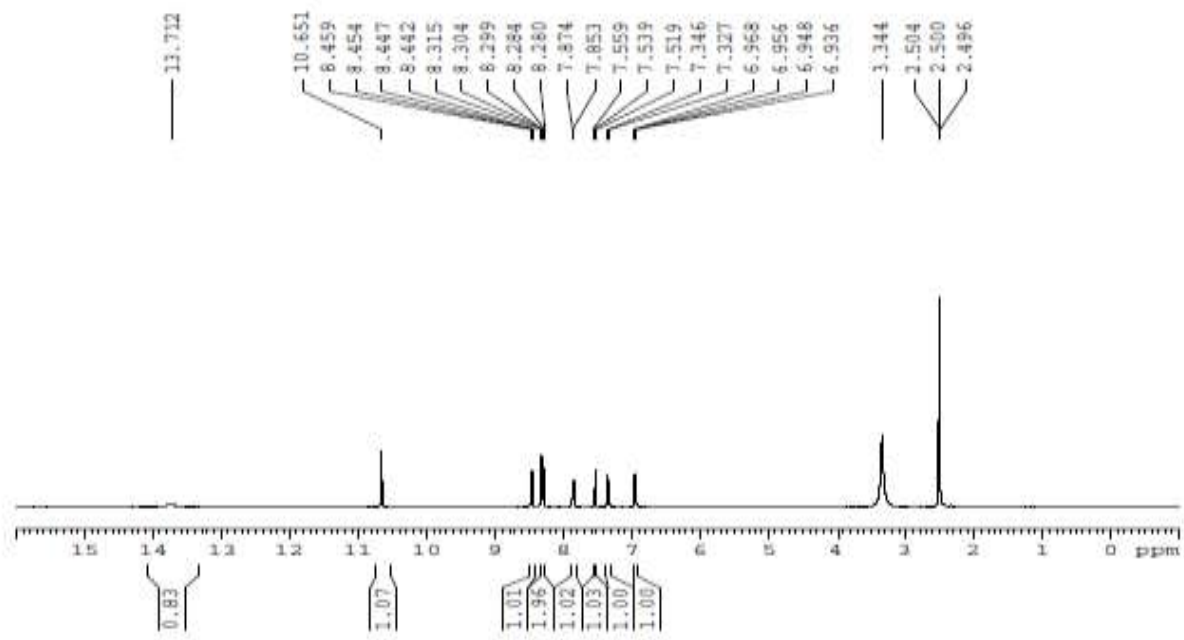

Figure-6:- Proton NMR Spectra of 2-((3-(trifluoromethyl)phenyl)amino)nicotinic acid in DMSO
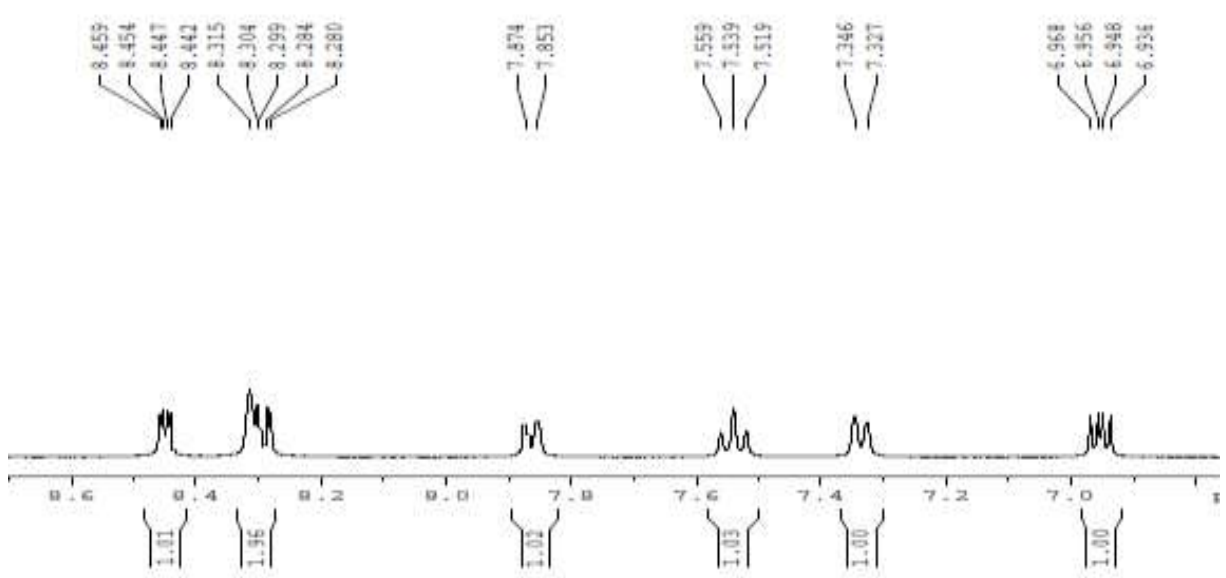

Figure-7:- Expanded view of Proton NMR Spectra of 2-((3- (trifluoromethyl)phenyl)amino)nicotinic acid in DMSO 


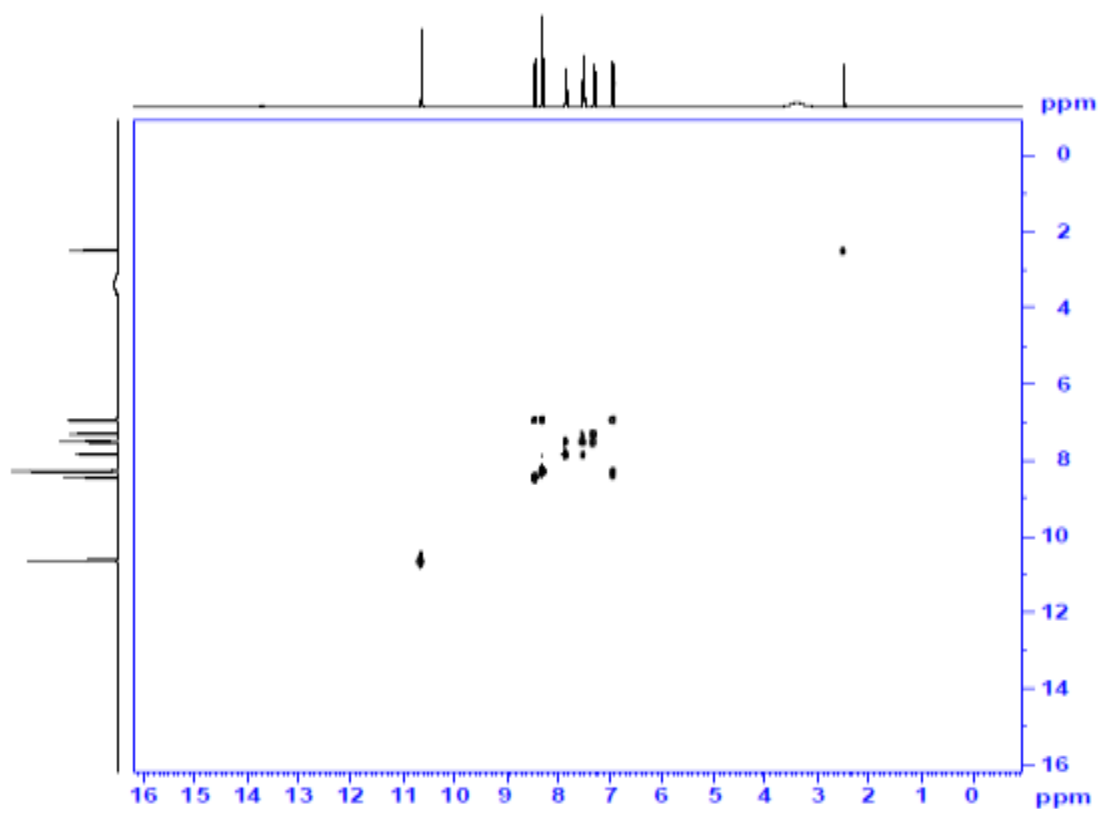

Figure-8:- COSY NMR Spectra of 2-((3-(trifluoromethyl)phenyl)amino)nicotinic acid in DMSO

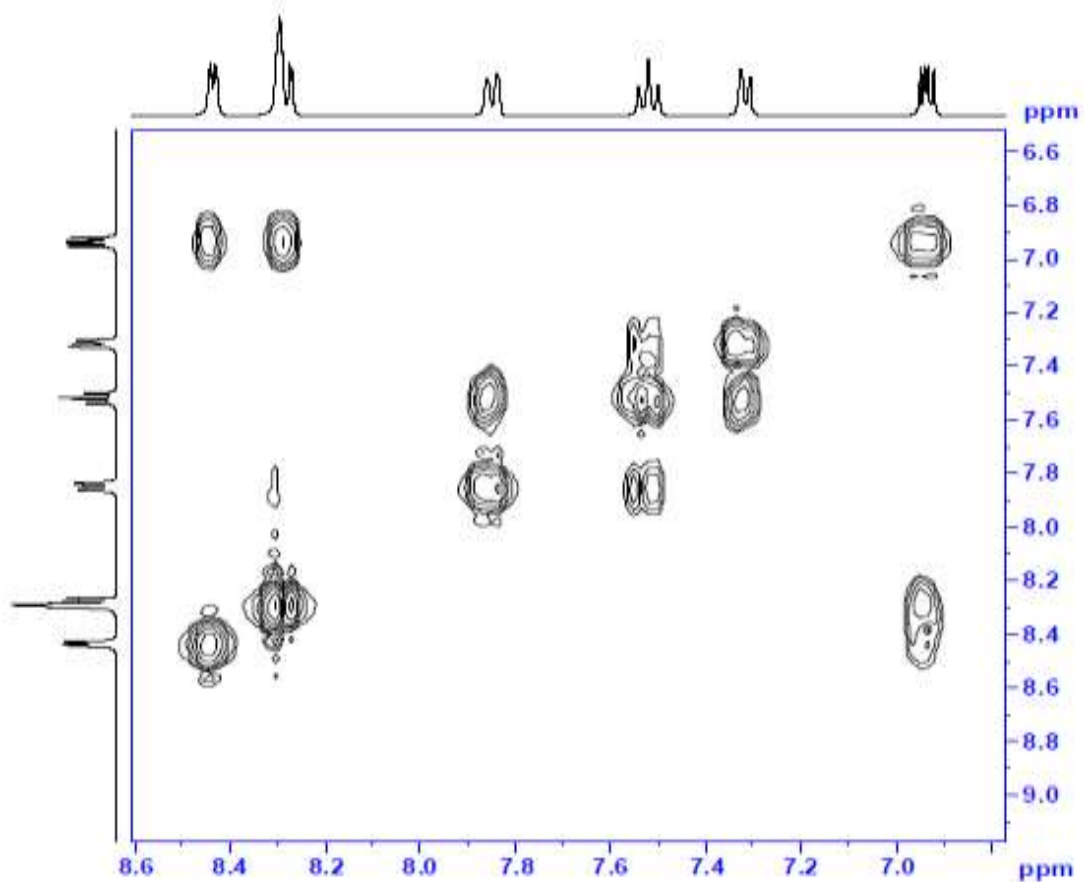

Figure-9:- Expanded View of COSY NMR Spectra of 2-((3-(trifluoromethyl)phenyl)amino)nicotinic acid in DMSO

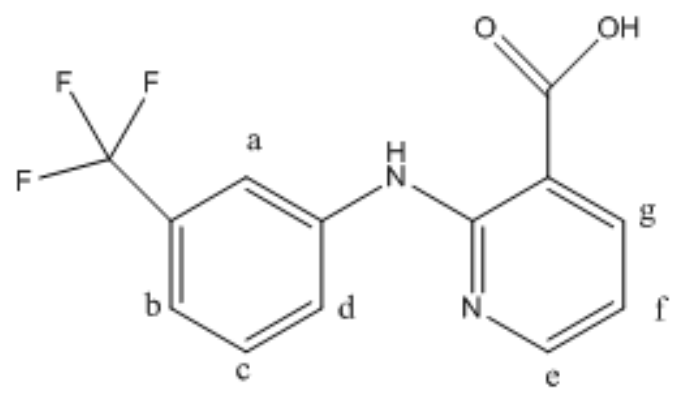

Figure-10:- Structure of 2-((3-(trifluoromethyl) phenyl)amino)nicotinic acid taken for study 
From the proton NMR data and COSY data we were able to elucidate all the peaks of the NMR spectra, the study concluded that acid peak is coming broad at $13.71 \mathrm{ppm}$ while $\mathrm{NH}$ is coming as sharp singlet at $10.65 \mathrm{ppm}$ proton ' $\mathrm{a}$ ' is also coming at $8.31 \mathrm{ppm}$ as singlet and is also merged with a doublet of doublet ( $8.30 \mathrm{ppm})$, rest of the protons are clear from COSY data analysis, proton 'f' is coming as triplet at $7.53 \mathrm{ppm}$ and is clearly showing correlation with ' $\mathrm{g}$ ' at $7.34 \mathrm{ppm}$ and ' $\mathrm{e}$ ' at $7.87 \mathrm{ppm}$ respectively, while proton ' $\mathrm{c}$ ' is coming as doublet of doublet at $6.95 \mathrm{ppm}$ and is clearly showing correlation with 'b' at $8.45 \mathrm{ppm}$ and proton 'c' at $8.31 \mathrm{ppm}$ respectively.

Thus Correlation spectroscopy proved to be one of the best tool for proton prediction when they are J-coupled.

\section{References}

[1] J. Jeener, Ampe`re International Summer School, Basko Polje, (former) Yugoslavia, 1971.

[2] W. P. Aue, E. Bartholdi and R. R. Ernst, J. Chem. Phys., 1976, 64, 2229-2246.

[3] J. N. S. Evans, Biomolecular NMR Spectroscopy, Oxford University Press, Oxford, 1995

[4] J. Cavanagh, W. J. Fairbrother, A. G. Palmer, N. J. Skelton and M. Rance, Protein NMR Spectroscopy:Principles and Practice, 2nd edition Academic Press (Elsevier), San Diego, 2006.

[5] J. Hahn, Higher-order 31P NMR spectroscopy of polyphosphorus compounds In: Phosphorus-31 NMR Spectroscopy in Stereochemical Analysis (Eds.: J.G. Verkade and L.D. Quin) VCH, Florida, 1987,pp. 331-364.

[6] D. J. States, R. A. Haberkorn and D. J. Ruben, J. Magn. Reson., 1982, 48, 286-292.

[7] M. Ohuchi, M. Hosono, K. Furihata and H. Seto, J. Magn. Reson., 1987, 72, 279-297.

[8] D. Marion and K. Wu"thrich, Biochem. Biophys. Res. Commun., 1983, 113,967-974.

[9] J. Keeler and D. Neuhaus, J. Magn. Reson., 1985, 63, 454-472.

[10] D. Marion, M. Ikura, R. Tschudin and A. Bax, J. Magn. Reson., 1989, 85, 393-399. 